\title{
HR AND BP VARIABILITY TO ASSESS AUTONOMIC TONE POST SPINAL ANESTHESIA
}

Alain Deschamps PhD MD, Jacqueline Nicolet MD, Ian Kaufman MD and Steven B Backman PhD MD

Department of Anesthesia of MUHC, Royal Victoria Hospital, 68 7Pine Avenue West, Montreal, Quebec, H3A 1A1

INTRODUCTION: Hemodynamic instability post spinal anesthesia results mainly from functional sympathectomy. Therefore, indices that can measure rapid and sudden changes in autonomic activity could help prevent hypotensive episodes. Analysis of HR and BP variability (HRV, BPV) by wavelet transform has been used successfully to evaluate the changes in parasympathetic and sympathetic tone following epidural analgesia, where changes in autonomic activity were not large enough to affect absolute values of $\mathrm{HR}$ and $\mathrm{BP}^{1}$. The goal of this study was to test whether the analysis of HRV and BPV by wavelet transform could estimate changes in autonomic activity produced by spinal anesthesia, which frequently produces hypotension.

METHODS: With hospital ethics approval, baseline ECG and continuous non-invasive BP were recorded for 16 min before, and 20 min after spinal anesthesia in 14 patients scheduled for elective cesarean section. The spinal contained $1.5 \mathrm{ml}$ of $0.75 \%$ bupivacaine, $25 \mathrm{mcg}$ of fentanyl, and $0.25 \mathrm{mg}$ of preservative free morphine, injected through a $27 \mathrm{G}$ spinal needle. Beat-to-beat R-R intervals and blood pressures were obtained for analysis HRV and BPV. Hypotension was treated with phenylephrine or ephedrine, as needed, and the time of the first and subsequent treatments were recorded. Analysis of HRV and BPV was obtained by Wavelet Transform every 2 min pre and post spinal. Low frequency power (LFP) and high frequency power (HFP) of BPV reflect changes in sympathetic activity, and HFP of HRV reflects parasympathetic activity ${ }^{1}$. A Repeated Measures ANOVA was used for statistical analysis, followed by a Dunnett Multiple Comparisons Test when significant. A $\mathrm{P}<0.05$ was considered significant.

RESULTS: LFP and HFP of BPV decreased post spinal anesthesia $(\mathrm{P}<0.02$ and $\mathrm{P}<0.005$, respectively). These indices of sympathetic tone were already decreased at 2 min post spinal anesthesia. HFP of HRV did not show significant increases post spinal anesthesia. Hypotension requiring treatment occurred in 13/14 patients. The time of the first treatment was $4.2 \pm 2.3 \mathrm{~min}($ mean $\pm \mathrm{SD})$, with a median of $4 \mathrm{~min}$ and a range of 2-9 $\min$.

DISCUSSION: Analysis of BP variability by wavelet transform could be used as an index of the changes in sympathetic outflow following spinal anesthesia for cesarean section.

REFERENCES: 1- Anesthesiology 2004; 101:21-7. 